\title{
Notalgia paréstesica; el paradigma del prurito neuropático. Descripción de seis casos
}

\author{
J.M. Gómez-Argüelles ${ }^{1}$, F. Michel ${ }^{2}$, L. Gómez Romero¹ y S. Somovilla ${ }^{2}$

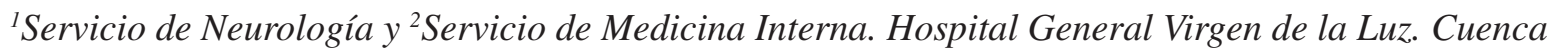

Gómez-Argüelles JM, Michel F, Gómez Romero L, Somovilla S. Notalgia paréstesica; el paradigma del prurito neuropático. Descripción de seis casos. Rev Soc Esp Dolor 2015; 22(5): 212-216.

\begin{abstract}
The notalgia paresthetica is a chronic sensory neuropathy, characterized by intense pain and/or itching in the upper or middle back, typically below the left shoulder. Usually associated hyperpigmentation area in the affected area, or close to it. Although the aetiology has not been established with certainty, has been explained by two reasons: either by a central degenerative process, or by a process of peripheral nerve entrapment at that level. There is not standardized treatment available, but is usually treated as any other process with neuropathic pain or itching. We present six typical cases of notalgia paresthetica, together with a review of the literature, with special emphasis on the different theories that try to explain the pathophysiology of this entity.
\end{abstract}

Key words: Neuropathic pain. Hyperpigmentation. Sensory neuropathy. Notalgia paresthetica. Itching.

\section{RESUMEN}

La notalgia parestésica es una neuropatía sensitiva crónica caracterizada por cursar con intenso dolor y/o prurito en la zona superior o media de la espalda, típicamente debajo del hombro izquierdo. Suele asociar una zona de hiperpigmentación en el área afectada o cercana a ella. Aunque la etiología no

Recibido: 04-07-14.

Aceptado: 08-09-14. se ha establecido con certeza, se ha intentado explicar por dos motivos, bien por un proceso degenerativo central, o bien por un cuadro de atrapamiento periférico de un nervio en ese ámbito. Aunque no se dispone de un tratamiento estandarizado, se suele tratar como cualquier otro tipo de proceso que curse con dolor o prurito neuropático. Se presenta una serie de seis casos de notalgia parestésica y se realiza una revisión de la literatura, haciendo especial hincapié en las diferentes teorías que intentan dilucidar la fisiopatología de esta entidad.

Palabras clave: Dolor neuropático. Hiperpigmentación. Neuropatía sensitiva. Notalgia parestésica. Prurito.

\section{INTRODUCCIÓN}

La notalgia parestésica (NP) es una neuropatía sensitiva poco conocida que se localiza en la espalda y que a menudo cursa con dolor y/o prurito. El término "notalgia" deriva de las palabras griegas notos, que significa "espalda", y algia, que significa "dolor". Esta condición fue descrita por primera vez en 1934 por Astwazaturow (1), quien ya supuso que se originaba por una lesión de una rama dorsal de los nervios espinales torácicos, entre T2 y T6. La clínica habitual es dolor de características neuropáticas, prurito neurógeno y/o parestesia en un área intermedia entre la escápula y la columna espinal torácica, casi siempre en la lado izquierdo, de forma unilateral, aunque puede ocurrir bilateral, y rara vez en el lado paravertebral derecho. Frecuentemente se observa además una lesión hiperpigmentada de la piel en la zona escapular. Se suele acompañar de alteraciones en la sensibilidad, tanto a estímulos tactiles térmicos como vibratorios, así como puede asociar hipoestesia al pinchazo. Se desconocen su etiología y prevalencia exactas (2). En el siguiente artículo describimos los casos 
de seis pacientes con diagnóstico de NP que acudieron a la consulta por dolor y/o molestias en la espalda de características principalmente pruriginosas.

\section{CASO CLÍNICO 1}

Mujer de 42 años que refiere que desde un año antes de acudir a la consulta presenta dolor tipo escozor en la zona del omóplato izquierdo y poco tiempo después observó que tenía una mancha hiperpigmentada en la espalda, en la zona dorsal media, pero que no coincidía con la zona pruriginosa. La sensación de picor era constante, a veces incluso con sensación de "descargas eléctricas". Le molestaba cualquier estímulo mecánico sobre esa zona, lo que le impedía llevar ropa ajustada e incluso evitaba cualquier mínimo roce. Se realizó una biopsia de la lesión hiperpigmentada, siendo compatible con el diagnóstico de notalgia parestésica (Fig.

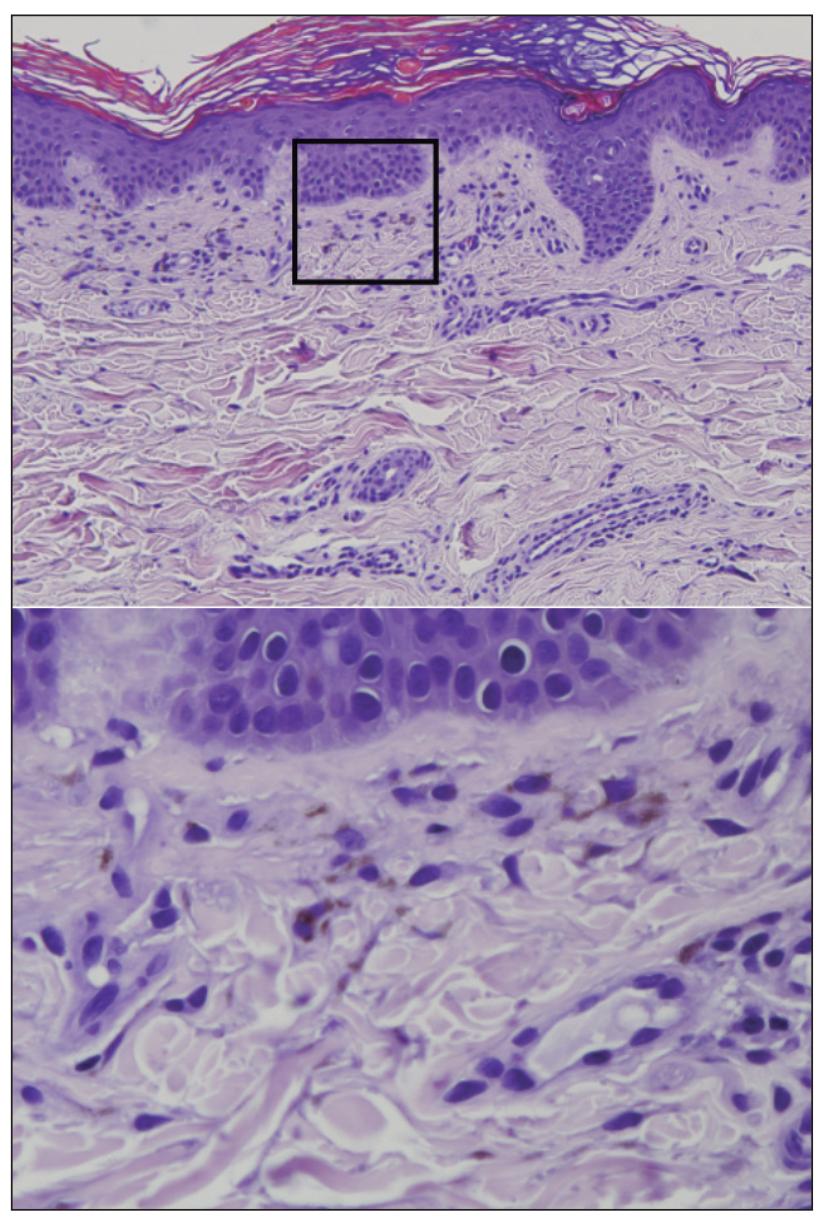

Fig. 1. La histología del caso 1 mostró una epidermis sin lesiones llamativas y una hipercelularidad de la dermis superior (HE X10). A mayor aumento (foto inferior), la hipercelularidad de la dermis está constituida por macrófagos cargados de pigmento melánico (melánofagos) (HE X40).
1). En la exploración se objetivaba una zona de hiperestesia en la región escapular izquierda, con alodinia cutánea y dinámica, más hiperestesia al pinchazo en una zona claramente delimitada. El estudio de resonancia magnética nuclear (RMN) de columna completa no mostró anomalías. Se intentaron diferentes tratamientos (ebastina, capsaícina en crema, corticoide tópico, pregabalina oral, lidocaína en parche al 5\%, capsaicina en parche al 8\%, infiltración con toxina botulínica), sin ninguna eficacia sobre los síntomas descritos y sin observarse mejoría de la clínica.

\section{CASO CLÍNICO 2}

Hombre de 56 años que, desde hace unos dos años, presenta molestias tipo parestesias (no dolor) en la espalda (región dorsal alta en el lado izquierdo) junto con mayor sensibilidad por el roce de la ropa en esa zona (hiperestesia mecánica). En la exploración se objetivaba una zona de hiperestesia en la región escapular izquierda, con hiperestesia cutánea y dinámica, una zona circular delimitada que no correspondía con la zona de hiperpigmentación (Fig. 2). La RMN de columna cervical y dorsal mostraba protrusiones discales en la zona de C4, C5, C6 y D1, sin afectación radicular, sin compromiso medular y con un estudio neurofisiológico normal. Se decidió no iniciar tratamiento, ya que al

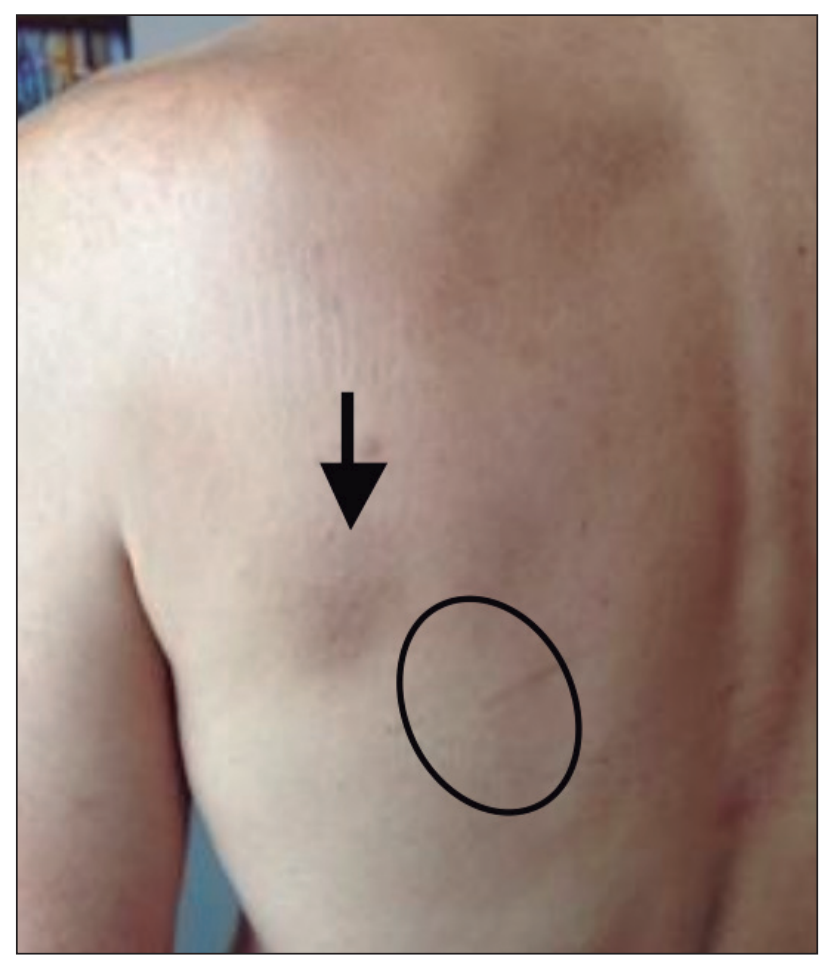

Fig. 2. NP del caso 2; situada en el lado izquierdo de la espalda del paciente (marcado con un óvalo el área de hiperestesia cutánea) y la placa de hiperpigmentación adyacente (flecha). 
paciente no le molestaban dichos síntomas y tampoco era partidario de ello.

\section{CASO CLÍNICO 3}

Mujer de 35 años que consulta por lesión pruriginosa en la espalda de dos años de evolución. Refiere prurito continuo en la región escapular derecha, pero no ha requerido tomar ningún tratamiento para ello porque lo describe como tolerable. En la exploración se objetiva, en la zona dorsal media, paravertebral, una zona de hiperpigmentación, y en un área más amplia (unos $2 \mathrm{~cm}$ alrededor de la zona hiperpigmentada) es donde se objetiva mayor hiperestesia al pinchazo en comparación con el resto de zonas. En las pruebas complementarias practicadas destaca una RMN de columna total que muestra una hernia discal central C5-C6 que contacta con la médula espinal sin desplazarla. No requirió tampoco tratamiento sintomático.

\section{CASO CLÍNICO 4}

Mujer de 51 años que presenta varias manchas cutáneas extensas en la región dorsal de espalda, de cuatro años de duración. Refiere sensación leve de picor en dichas zonas, sobre todo en épocas de calor. Se le practicó biopsia de piel de una de ellas, siendo compatible con un infiltrado crónico perivascular junto a melanófagos en la dermis superficial y leve hiperpigmentación de la capa basal de la epidermis. Fue Rojo Congo negativo. No se pudo practicar RMN por padecer claustrofobia. No requirió tratamiento.

\section{CASO CLÍNICO 5}

Mujer de 56 años que presenta una lesión maculosa adyacente a la columna, en la zona de D5-D7, levemente pruriginosa, de dos años de evolución. En la exploración neurológica, como única alteración presentaba una hiperestesia al pinchazo justo en el área de la lesión. Se le realizó una RMN de columna dorsal que fue normal. Tampoco requirió tratamiento farmacológico.

\section{CASO CLÍNICO 6}

Mujer de 45 años que consulta por un cuadro de cuatro años de duración de prurito en dos zonas paravertebrales diferenciadas, en la zona de D2 y D7, paravertebrales ambas. Aumentaba el prurito solo en época de mayor calor. La exploración fue normal, así como la RMN de columna practicada. Se intentó tratamiento con capsaicina tópica, pero como la paciente no lo toleraba y en cambio sí el prurito, prefirió no probar otros tratamientos farmacológicos.

\section{DISCUSIÓN}

La NP constituye una entidad enigmática de la que no se conocen aún bien su prevalencia ni su fisiopatología, ni dispone de un tratamiento completamente estandarizado. Se sospecha que es una enfermedad relativamente rara (3), pero no existen estudios prospectivos para verificar esta aseveración. Ocurre con mayor frecuencia en mujeres de media edad o ancianos (2), pero puede ser en jóvenes en los pocos casos hereditarios descritos. Se engloba dentro de las mononeuropatías sensitivas focales, de las que se han descrito diferentes territorios neurocutáneos tales como el prurito braquiorradial (nervio cutáneo anterobraquial), la quiralgia parestésica (nervio radial), la meralgia parestésica (nervio femorocutáneo), la gonalgia parestésica (nervio safeno) o la digitalgia parestésica (nervio digital), entre otras (4). Sobre su origen se han postulado diversas teorías, siendo las más extendidas las dos siguientes:

TABLA I. DATOS DE LOS PACIENTES REGISTRADOS

\begin{tabular}{lccccccc}
\hline \multicolumn{1}{c}{ Caso } & Sexo & Edad & Años $^{*}$ & Metámeras & Imagen $^{* *}$ & Biopsia & Tratamiento \\
\hline $1 .{ }^{\circ}$ & $\mathrm{M}$ & 42 & 2 & $\mathrm{D} 4-\mathrm{D} 6$ & $\mathrm{~N}$ & Sí & Sí \\
$2 .^{\circ}$ & $\mathrm{V}$ & 56 & 2 & $\mathrm{D} 7-\mathrm{D} 8$ & $\mathrm{~A}$ & No & No \\
$3 .^{\circ}$ & $\mathrm{M}$ & 35 & 1 & $\mathrm{D} 5$ & $\mathrm{~A}$ & No & No \\
$4 .{ }^{\circ}$ & $\mathrm{M}$ & 51 & 4 & D2-D4, D7,D8 & - & Sí & No \\
$5 .^{\circ}$ & $\mathrm{M}$ & 56 & 2 & D5-D7 & $\mathrm{N}$ & No & No \\
$6 .{ }^{\circ}$ & $\mathrm{M}$ & 45 & 4 & D2 y D7-D8 & $\mathrm{N}$ & No & No \\
\hline
\end{tabular}

M: mujer. V: varón. N: normal. A: anormal.

*Años de evolución hasta el diagnóstico de la NP.

** Estudio de RMN de columna dorsal realizado. 
1. Esta entidad está producida por un proceso vertebral degenerativo, traumático o compresivo, como por ejemplo una herniación del núcleo pulposo o un osteofito $(3,5)$. Savk y cols. (6) describen que en un $80 \%$ de sus pacientes existían alteraciones radiológicas en la médula espinal y en un $60-70 \%$ de ellos estas anormalidades se correlacionaban con la localización topográfica de la NP. Según estos autores, la compresión de una raíz nerviosa por una columna con un proceso degenerativo sería la causa de esta entidad. Sin embargo, otros estudios con mayor número de pacientes encuentran una baja correlación entre las alteraciones degenerativas o compresivas de la médula espinal con la localización de la NP (7). Estos mismos autores piden precaución al interpretar las alteraciones radiológicas con esta entidad, ya que hasta la mitad de los voluntarios sanos tienen alteraciones radiológicas en la médula dorsal sin presentar ningún otro problema (8), siendo claramente estos cambios dependientes de la edad.

2. La otra hipótesis en la etiología se focaliza en una alteración en la inervación de las ramas posteriores de los nervios periféricos espinales (9). Pleet y cols. (2) sugieren que se debe a la peculiaridad de los ramos posteriores de los nervios espinales que salen de T2-T6, porque al atravesar el músculo paraespinal multifidus cambia su dirección $90^{\circ}$, lo que los predispone a atrapamientos, lo que conlleva posteriormente cambios isquémicos y, en último término, lesión del nervio. En algunos casos los nervios lesionados se pueden "encender" espontáneamente, produciendo dolor o prurito y, consecuentemente, alterar la neuroanatomía de la piel. Wang y cols. (10) objetivaron que una lesión de un nervio torácico por disfunción del músculo serrato anterior era la causa de NP en cuatro pacientes. En algún caso se ha demostrado incluso denervación paraespinal por medio de electromiografía, lo que sugiere compresión nerviosa (11).

En cuanto a la clínica, la localización es casi siempre entre los segmentos dorsales T2 y T8 (regiones interescapular, subescapular o paravertebral). Se ha descrito afectación de regiones inferiores (5), pero estos casos son probablemente radiculopatías toracolumbares o intercostales. Lo habitual es que curse con dolor de características neuropáticas, o más frecuentemente con prurito (hasta en un $60 \%$ de los casos) (7), que puede considerarse también de tipo neuropático. Comparado con el dolor neuropático, el prurito es menos reconocido como secuela de una lesión nerviosa (12). Por ejemplo, el prurito es un síntoma presente en más del $40 \%$ de los pacientes con neuralgia postherpética (13). Se cree que el dolor neuropático puede tener una patogenia similar al prurito (14). La estimulación de fibras $\mathrm{C}$ y A-delta puede directamente producir prurito, $\mathrm{o}$ el prurito puede resultar indirectamente de la degranulación de los mastocitos, causado por la sustancia $\mathrm{P}(14,15)$. No es raro que muchos clínicos no reconozcan la naturaleza neuropática de este tipo de prurito (16).

Respecto al origen de la lesión hiperpigmentada circunscrita, en forma de mácula, que se observa con frecuencia en la NP (en dos terceras partes de los casos publicados), también se ha especulado bastante. Podría ser una consecuencia directa de la disfunción neurológica, pensando que la liberación de la sustancia $\mathrm{P}$ puede producir proliferación de keratocitos, células musculares lisas arteriales y fibroblastos (14). Alternativamente, se piensa que la hiperpigmentación puede ser producida por el propio rascado del prurito (10), con el consiguiente crecimiento keranocítico. Nosotros opinamos que es más factible la primera explicación, ya que la zona de hiperpigmentación no suele corresponderse con la zona de prurito, como ocurrió en dos de nuestros casos. Los casos en los que se ha practicado biopsia suelen mostrar hiperpigmentación epidérmica o dérmica, con keratinocitos necróticos intraepiteliales y melanófagos en la dermis papilar. Depósitos de amiloide solo se encuentran en menos del 10\% de las biopsias (7).

Aunque la evolución de la NP es benigna y su tratamiento es puramente sintomático, casi siempre tiene una eficacia parcial o transitoria. El único medicamento sometido a ensayo clínico ha sido la capsaicina tópica al 0,0025\%, con resultados iniciales buenos pero que empeoraban al suspender la aplicación del tratamiento (17). Se han utilizado diversos tratamientos, aunque suele ser en casos aislados, con mayor o menor éxito, tales como el bloqueo anestésico paravertebral, fisioterapia, antihistamínicos, capsaicina al 8\%, antiepilépticos, antidepresivos, corticoides, toxina botulínica, TENS, etc. Ninguno puede ser considerado el tratamiento estándar, lo que hace difícil decidir cuál utilizar como mejor opción en cada caso (18). En nuestro primer caso ninguno de los tratamientos propuestos fue eficaz, ni siquiera parcialmente, lo que corrobora la refractariedad de algunos casos con esta patología, mientras que en los otros casos no fue necesario.

Por tanto, aunque no se sabe a ciencia cierta la prevalencia de esta entidad, la NP debe ser reconocida como una neuropatía sensitiva que cursa con dolor o prurito, ambos de origen neuropático, y que consideramos se origina como consecuencia de una lesión de un nervio periférico espinal dorsal. Su tratamiento no está establecido, por lo que se debe tener en cuenta su fisiopatología para establecer la mejor opción terapéutica en cada caso.

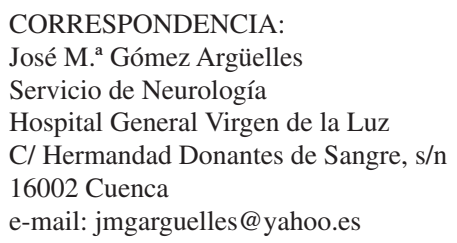




\section{BIBLIOGRAFÍA}

1. Astwazaturow M. Uber parästhetische Neuralgien und eine besondere Form derselben-Notalgia paresthetica. Dtsch Z Nerven Heilkd 1934;133:188-96.

2. Pleet AB, Massey EW. Notalgia paresthetica. Neurology 1978;28:1310-12.

3. Savk E, Savk O, Bolukbasi O, Culhaci N, Dikicioglu E, Karaman G, et al. Notalgia paresthetica: a study on patogenesis. Int J Dermatol 2000;39:754-9.

4. Massey EW. Sensory mononeuropathies. Semin Neurol 1998;18:177-83.

5. Raison-Peyron N, Meunier L, Acevedo M, Meynadier J. Notalgia paresthetica: clinical, physiopathological and therapeutic aspects. A study of 12 cases. J Eur Acad Dermatol Venereol 1999;12:215-21.

6. Savk O, Savk E. Investigation of spinal pathology in notalgia paresthetica. J Am Acad Dermatol 2005;52:1085-7.

7. Huesmann T, Cunha PR, Osada N, Huesmann M, Zanelato TP, Phan NQ, et al. Notalgia paraesthetica: a descriptive two-cohort study of 65 patients from Brazil and Germany. Acta Derm Venereol 2012;92(5):535-40.

8. Matsumoto M, Okada E, Ichihara D, Watanabe K, Chiba $\mathrm{K}$, Toyama Y, et al. Age-related changes of thoracic and cervical intervertebral discs in asymptomatic subjects. Spine 2010;35:1359-64.

9. Streib EW, Sun SF. Notalgia paresthetica owing to compression neuropathy: case presentation including electrodiagnostic studies. Eur Neurol 1981;20:64-7.
10. Wang CK, Gowda A, Barad M, Mackey SC, Carroll IR. Serratus muscle stimulation effectively treats notalgia paresthetica caused by long thoracic nerve dysfunction: a case series. J Brachial Plex Peripher Nerve Inj 2009;4:17.

11. Massey EW, Pleet AB. Electromyographic evaluation of nostalgia paresthetica. Neurology 1981;31:642.

12. Henderson J, Terenghi G, McGrouther DA, Ferguson MW. The reinnervation pattern of wounds and scars may explain their sensory symptoms. J Plast Reconstr Aesthet Surg 2006;59(9):942-50.

13. Oaklander AL, Bowsher D, Galer B, Haanpää M, Jensen MP. Herpes zoster itch: preliminary epidemiologic data. J Pain 2003;4(6):338-43.

14. Weidner C, Klede M, Rukwied R, Lischetzki G, Neisius U, Skov PS, et al. Acute effects of substance $\mathrm{P}$ and calcitonin gene-related peptide in human skin - a microdialysis study. J Invest Dermatol 2000;115(6):1015-20.

15. Tanaka T, Danno K, Ikai K, Imamura S. Effects of substance $\mathrm{P}$ and substance $\mathrm{K}$ on the growth of cultured keratinocytes. J Invest Dermatol 1988;90(3):399-401.

16. Oaklander AL. Neuropathic itch. Semin Cutan Med Surg 2011;30(2):87-92.

17. Wallengren J, Klinker M. Successful treatment of notalgia paresthetica with topical capsaicin: vehicle-controlled, double-blind, crossover study. J Am Acad Dermatol 1995;32:287-9.

18. Richardson BS, Way BV, Speece AJ. Osteopathic manipulative treatment in the management of notalgia paresthetica. J Am Osteopath Assoc 2009;109(11):605-8. 